\title{
Precancerous cervical lesion in Ethiopia: systematic review and meta-analysis
}

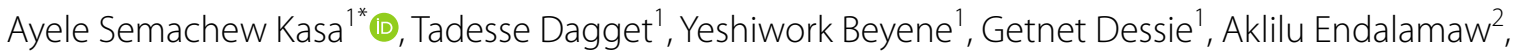 \\ Yinager Workineh², Emiru Ayalew ${ }^{1}$, Balew Zeleke ${ }^{2}$, Sitotaw Kerie ${ }^{1}$, Getasew Mulat ${ }^{3}$ and Worku Animaw ${ }^{1}$
}

\begin{abstract}
Background: Though cervical cancer is largely preventable, it is still the second most common female cancer globally and the leading cause of cancer deaths among females in African. Though many efforts have been done to study the burden of the disease in Ethiopia, primary studies examining the prevalence of precancerous cervical lesions are fragmented. Hence, this systematic review and meta-analysis is aimed at estimating the pooled prevalence of precancerous cervical lesion and its trends in Ethiopia.

Methods: This systematic review and meta-analysis was conducted using the following electronic databases. PubMed, Web of Science, SCOPUS, Science Direct, Google Scholar, African Index Medicus (AIM), African Journals Online databases, and Addis Ababa and Bahir Dar Universities research repositories were searched following the Preferred Items for Systematic Review and Meta-analysis (PRISMA) Guideline. STATA 15 statistical software was used to analyze the data. The quality of the included studies was assessed using the Joanna Briggs Institute (JBI) quality appraisal tool for meta-analysis. Heterogeneity between studies was assessed using the Cochrane $Q$ test and $P$ test statistics based on the random effects model. A random effects model was computed to estimate the pooled prevalence of precancerous cervical lesion in Ethiopia. Finally, the trend of precancerous cervical lesion in the country was presented.
\end{abstract}

Result: Seventeen studies with a total of 26,112 participants were included in the analysis. The pooled prevalence of precancerous cervical lesion was 15.16 (95\% Cl 10.16-19.70). The subgroup analysis by region showed the highest prevalence of precancerous cervical lesion at the Southern Nations and Nationalities Peoples Region (19.65\%; $95 \% \mathrm{Cl}$ 15.51-23.80). The trend of precancerous cervical lesion prevalence showed an increased pattern over time.

Conclusion: Approximately one among six of the study participants had precancerous cervical lesion. The trend also showed that there is still an increasing precancerous cervical lesion in Ethiopia. Best practices in achieving high vaccination coverage shall be informed by practices in other successful countries.

Keywords: Cervical cancer, Cervical lesion, Precancerous, Systematic review, Ethiopia

\section{Background}

Cancers that originate in the female reproductive system are called women's reproductive cancers. These include cancer of the cervix, breast, ovaries, vagina, vulva, and endometrium [1-3]. Though cervical cancer (CC) is

*Correspondence: finoteayu24@gmail.com; ayele.semachew@yahoo.com ${ }^{1}$ Department of Adult Health Nursing, College of Medicine and Health Sciences, Bahir Dar University, Bahir Dar, Ethiopia

Full list of author information is available at the end of the article largely preventable, it is still the second most common female cancer internationally and the leading cause of cancer deaths among females in African countries [4, 5]. Low levels of awareness and lack of effective screening programs, overshadowed by other communicable health priorities, are the possible factors for the observed higher incidence rate of cervical cancer in the developing regions of the world $[1,6]$.

Every year, approximately 500,000 new CC cases are diagnosed and 270,000 women die of this disease, mostly original author(s) and the source, provide a link to the Creative Commons licence, and indicate if changes were made. The images or other third party material in this article are included in the article's Creative Commons licence, unless indicated otherwise in a credit line to the material. If material is not included in the article's Creative Commons licence and your intended use is not permitted by statutory regulation or exceeds the permitted use, you will need to obtain permission directly from the copyright holder. To view a copy of this licence, visit http://creativecommons.org/licenses/by/4.0/. The Creative Commons Public Domain Dedication waiver (http://creativeco mmons.org/publicdomain/zero/1.0/) applies to the data made available in this article, unless otherwise stated in a credit line to the data. 
in developing countries [7, 8]. Globally, $86 \%$ of all CC diagnosed and $88 \%$ of $\mathrm{CC}$ deaths occur in developing regions of the world [9]. Cervical cancer is the second most frequently diagnosed cancer and the leading cause of cancer death in African women [10,11].

The incidence is growing, as an estimated 570,000 new cases of CC was recorded in 2018, with approximately $90 \%$ of the deaths occurring in low- and middle-income countries [12]. Rates vary substantially across regions, with the incidence and death rates in East Africa and West Africa similar to rates in North Africa [13].

Reports of trends in CC mortality from less developed countries have been limited by poor data quality and inaccurate population estimates [14]. But because of poor access to quality screening and treatment service, the trend is increasing in these countries. According to trend analysis on CC between the years 1980 and 2010, the CC incidence rate was increased from 378,000 to 454,000 [15]. By 2020, it has been estimated that CC will be diagnosed in over 665,035 women worldwide, and 357,852 will die as a result [8].

In Africa, which has a population of 267.9 million women aged 15 years and older at risk of developing cervical cancer, approximately 80,000 women are diagnosed with cervical cancer per year, and just more than 60,000 women die from the disease [16]. Of the African burden of CC, the incidence and mortality in Sub-Saharan Africa are among the highest in the world and account for over $70 \%$ of the global CC burden with 70,000 new cases annually $[8,17]$.

According to the 2009 World Health Organization (WHO) report, the age-adjusted incidence rate of cervical cancer in Ethiopia was 35.9 per 100,000 patients with 7619 annual number of new cases and 6081 deaths every year [3, 18-20]. Other studies also showed that, of the nearly 22 million Ethiopian women over the age of 15 , approximately 7600 are diagnosed with cervical cancer and roughly 6000 women die of the disease each year [18, 21-23].

Though many efforts have been done to study the burden of precancerous cervical lesion, primary studies reporting prevalence studies are fragmented. Previous studies in the literature have shown variations and discrepancies across different studies in Ethiopia. Hence, this systematic review and meta-analysis aims to consolidate data regarding the prevalence of precancerous cervical lesion in Ethiopia.

\section{Methods \\ Reporting}

The Preferred Reporting Items of Systematic Reviews and Meta-Analysis (PRISMA) checklist guideline was used to report the result of this systematic review and meta-analyses. In addition, the PRISMA flow chart was utilized to show the selection process of studies for a systematic review and meta-analysis [24].

\section{Searching strategies}

This systematic review and meta-analysis was conducted to estimate the pooled prevalence of precancerous cervical lesion in Ethiopia. To conduct this study, all potentially relevant articles and gray literatures were meticulously searched. PubMed, Web of Science, SCOPUS, Science Direct, Google Scholar, African Index Medicus (AIM) and African Journals Online databases, and Addis Ababa and Bahir Dar Universities research repositories were searched using the following search terms: "Epidemiology," "Burden," "Magnitude," "Prevalence," "Cervical cancer," "Cervix neoplasm," "Cervix lesion," "Pap Smear Positive," "VIA Positive," "Cervix precancerous lesion," and "Ethiopia." Search strings were developed using "AND" and "OR" Boolean operators. In addition to this, gray literature studies were searched from a research repository online library and a secondary search technique known as "footnote chasing" was utilized to identify additional articles from the included articles.

The search was carried out between August 28 and October 10, 2019, and all articles published until October 10,2019 , were included in the review.

\section{Inclusion and exclusion criteria}

This systematic review and meta-analysis encompassed studies conducted only in Ethiopia using the English language. Research articles published in scientific journals and gray literature that reported the prevalence of precancerous cervical lesion were included in the review. Excluded studies included any which focused on the assessment of knowledge, attitude, and practice towards CC without the outcome of interest of this study, program evaluation studies, studies with only abstracts, case studies, and qualitative studies.

\section{Data extraction}

Three authors (ASK, TD, and YB) extracted all necessary data by a standardized data extraction format using Microsoft Excel. The extracted parameters were primary author, publication year, region where the study was conducted, method of assessment, study design, mean age of the study participants, response rate, sample size, and prevalence of precancerous cervical lesion. Then, three authors (GD, AE, and YW) checked the data extraction process. Finally, five authors (BZ, EA, SK, GM, and WA) participated in approving the extraction process. 


\section{Quality of the included studies}

To appraise the quality of the included articles, we used the Joanna Briggs Institute (JBI) quality appraisal tool adapted for studies reporting prevalence data [25]. The following items were used to appraise cross-sectional studies: (1) inclusion criteria, (2) description of study subject and setting, (3) valid and reliable measurement of exposure, (4) objective and standard criteria used, (5) identification of confounder, (6) strategies to handle confounder, (7) outcome measurement, and (8) appropriate statistical analysis. Studies were considered low risk whenever fitted to $50 \%$ and/or above in the quality assessment checklist criteria [25, 26]. Using the aforementioned quality appraisal tools, no study was excluded (Table 1).

\section{Data analysis}

The data were analyzed using STATA version 15 Statistical Software. Heterogeneity across studies was checked using the inverse variance $\left(I^{2}\right)$ and Cochran $Q$ statistics. The cut-offs of $25 \%, 50 \%$, and $75 \%$ were used to declare the heterogeneity as low, moderate, and severe, respectively [44, 45].

As the preliminary output of the test statistics revealed a significant heterogeneity among studies $\left(I^{2}>70 \%, P<\right.$ $0.05)$, a random effects model was used to estimate the pooled prevalence of precancerous cervical lesion with a 95\% confidence interval (CI). Subgroup analysis was also performed based on study area (regions) and human immunodeficiency virus (HIV) status in relation to the outcome variable. Funnel plot, Egger, and Begg tests at $5 \%$ significant level were employed to assess publication bias $[46,47]$.

\section{Results}

\section{Description of the identified studies}

Up to October 10, 2019, we identified a total of 1139 articles using different databases, Addis Ababa and Bahir Dar University research repositories. Nine hundred twenty-four articles were excluded because of duplication and relevance-related issues. Among the remaining 215 articles, 183 articles were excluded because the outcome was not clearly measured. Fifteen articles were excluded because they were program evaluation and knowledge, attitude, and practice-related reports. Finally, 17 studies fulfilled the eligibility criteria and were included in the final systematic review and meta-analysis (Fig. 1).

\section{Overview of included studies}

Among the total 17 studies included in this current review, five of them were from Addis Ababa City Administration [29, 35-37, 40]. Five studies were

Table 1 Critical appraisal result of the included studies, 2019

\begin{tabular}{|c|c|c|c|c|c|c|c|c|c|}
\hline \multirow[t]{2}{*}{ Included articles } & \multicolumn{9}{|c|}{ Criterion no. (items included to appraise cross-sectional and case-control studies) } \\
\hline & 1 & 2 & 3 & 4 & 5 & 6 & 7 & 8 & $\%$ \\
\hline Awoke et al. [27] & $\checkmark$ & $\checkmark$ & $\checkmark$ & $\checkmark$ & $\checkmark$ & $\checkmark$ & $\checkmark$ & $\checkmark$ & 100 \\
\hline Belayneh et al. [28] & $\checkmark$ & $\checkmark$ & $\checkmark$ & $x$ & $\checkmark$ & $\checkmark$ & $x$ & $\checkmark$ & 75 \\
\hline Birra [29] & $\checkmark$ & $\checkmark$ & $\checkmark$ & $x$ & $\checkmark$ & $\checkmark$ & $\checkmark$ & $\checkmark$ & 87.5 \\
\hline Gedefaw et al. [30] & $\checkmark$ & $\checkmark$ & $\checkmark$ & $\checkmark$ & $\checkmark$ & $\checkmark$ & $\checkmark$ & $\checkmark$ & 100 \\
\hline Getinet et al. [31] & $\checkmark$ & $\checkmark$ & $\checkmark$ & $\checkmark$ & $\checkmark$ & $\checkmark$ & $\checkmark$ & $\checkmark$ & 100 \\
\hline Hailemariam et al [32] & $\checkmark$ & $\checkmark$ & $\checkmark$ & $\checkmark$ & $\checkmark$ & $x$ & $\checkmark$ & $\checkmark$ & 87.5 \\
\hline Kassa et al. [33] & $\checkmark$ & $\checkmark$ & $\checkmark$ & $\checkmark$ & $\checkmark$ & $\checkmark$ & $\checkmark$ & $\checkmark$ & 100 \\
\hline Kebede et al. [34] & $\checkmark$ & $\checkmark$ & $\checkmark$ & $\checkmark$ & $\checkmark$ & $\checkmark$ & $\checkmark$ & $\checkmark$ & 100 \\
\hline Meseret and Tadiwos [35] & $\checkmark$ & $\checkmark$ & $\checkmark$ & $\checkmark$ & $\checkmark$ & $\checkmark$ & $\checkmark$ & $\checkmark$ & 100 \\
\hline Netsanet et al. [36] & $\checkmark$ & $\checkmark$ & $\checkmark$ & $\checkmark$ & $\checkmark$ & $\checkmark$ & $\checkmark$ & $\checkmark$ & 100 \\
\hline Pelzer et al. [37] & $\checkmark$ & $x$ & $\checkmark$ & $\checkmark$ & $x$ & $x$ & $\checkmark$ & $\checkmark$ & 62.5 \\
\hline Ruland et al. [38] & $\checkmark$ & $\checkmark$ & $\checkmark$ & $\checkmark$ & $x$ & $x$ & $\checkmark$ & $\checkmark$ & 75 \\
\hline Sami-Ramzi et al. [39] & $\checkmark$ & $\checkmark$ & $\checkmark$ & $\checkmark$ & $\checkmark$ & $\checkmark$ & $\checkmark$ & $\checkmark$ & 100 \\
\hline Teame et al. [40] & $\checkmark$ & $\checkmark$ & $\checkmark$ & $\checkmark$ & $\checkmark$ & $\checkmark$ & $x$ & $\checkmark$ & 90 \\
\hline Teka et al. [41] & $\checkmark$ & $\checkmark$ & $\checkmark$ & $\checkmark$ & $\checkmark$ & $\checkmark$ & $\checkmark$ & $\checkmark$ & 100 \\
\hline Temesgen et al. [42] & $\checkmark$ & $\checkmark$ & $\checkmark$ & $\checkmark$ & $\checkmark$ & $\checkmark$ & $\checkmark$ & $\checkmark$ & 100 \\
\hline Zewdie et al. [43] & $\checkmark$ & $\checkmark$ & $\checkmark$ & $\checkmark$ & $\checkmark$ & $\checkmark$ & $\checkmark$ & $\checkmark$ & 100 \\
\hline
\end{tabular}

$\sqrt{ }$ criterion fulfilled, $X$ criterion not fulfilled

Criterion no. 1: inclusion criteria, criterion no. 2: description of study subject and setting, criterion no. 3: valid and reliable measurement of exposure, criterion no. 4: objective and standard criteria used, criterion no. 5: identification of confounder, criterion no. 6: strategies to handle confounder, criterion no. 7: outcome measurement, and criterion no. 8: appropriate statistical analysis 


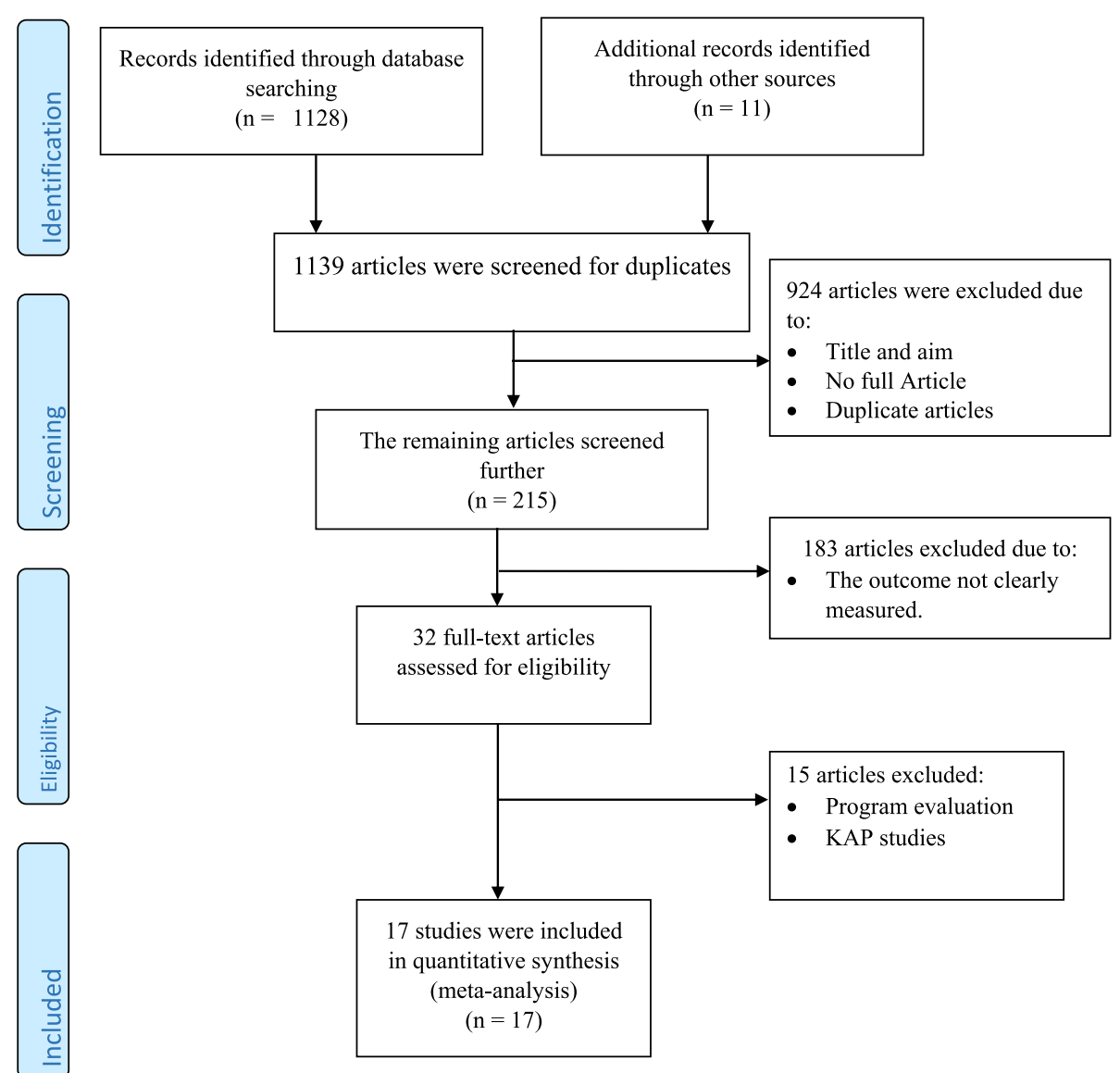

Fig. 1 PRISMA flow diagram showing the selection of studies for a systematic review and meta-analysis, 2019

included from the Southern Nations Nationalities and People Region (SNNPR) [30, 32, 38, 39, 41]. In addition, five studies were included from the Amhara region [27, $28,31,33,42]$. The remaining two articles were from Oromia [43] and Tigray [34]. Most of the studies used VIA as the measure of assessment for the outcome variable. The total participants in the included studies were 26,112 with samples ranging from 189 [38] to 16,632 [36]. The response rate of all the included studies was greater than $90 \%$, and the quality score of the studies ranged from 62.5 to $100 \%$ (Table 2).

\section{Publication bias}

To assess publication bias, both the funnel plot and the Egger's test were conducted in the meta-analysis. The visual examination of the funnel plot exhibited a symmetric distribution of studies (Fig. 2). In addition to the funnel plot, Egger's regression test was $(\beta=-0.0063$, $S E=0.08, P=0.88$ ) showing no statistical evidence of publication bias for the included studies.

\section{Prevalence of precancerous cervical lesion}

The lowest prevalence of precancerous cervical lesion was $1.56 \%$ which is reported from SNNPR [37] whereas the highest prevalence was $28.4 \%$ [29] reported from a study done in Addis Ababa. Overall, the pooled prevalence of precancerous cervical lesion in Ethiopia was 15.16 (95 CI 10.16-19.70) (Fig. 3).

$I^{2}$ statistic test for heterogeneity indicated that the studies differed significantly $\left(I^{2}=95.40 \%, p<0.001\right)$. Hence, we used a DerSimonian and Laird random effects model $[48,49]$ to estimate the pooled prevalence of precancerous cervical lesion. Studies that showed the largest weight were Plezer et al. [37] that showed 6.35, Kebede et al. [34] with 6.11, Temesgen et al. [42] with 6.10, and Belayneh et al. [28] that showed 6.80 weight, whereas Birara et al. showed a relatively smaller weight which was 5.62 (Fig. 3).

Despite having one of the lowest single study prevalence rates, the highest pooled subgroup analysis of precancerous cervical lesion prevalence was found in SNNPR, 19.65\% (95\% CI 15.51-23.80), followed by Addis 


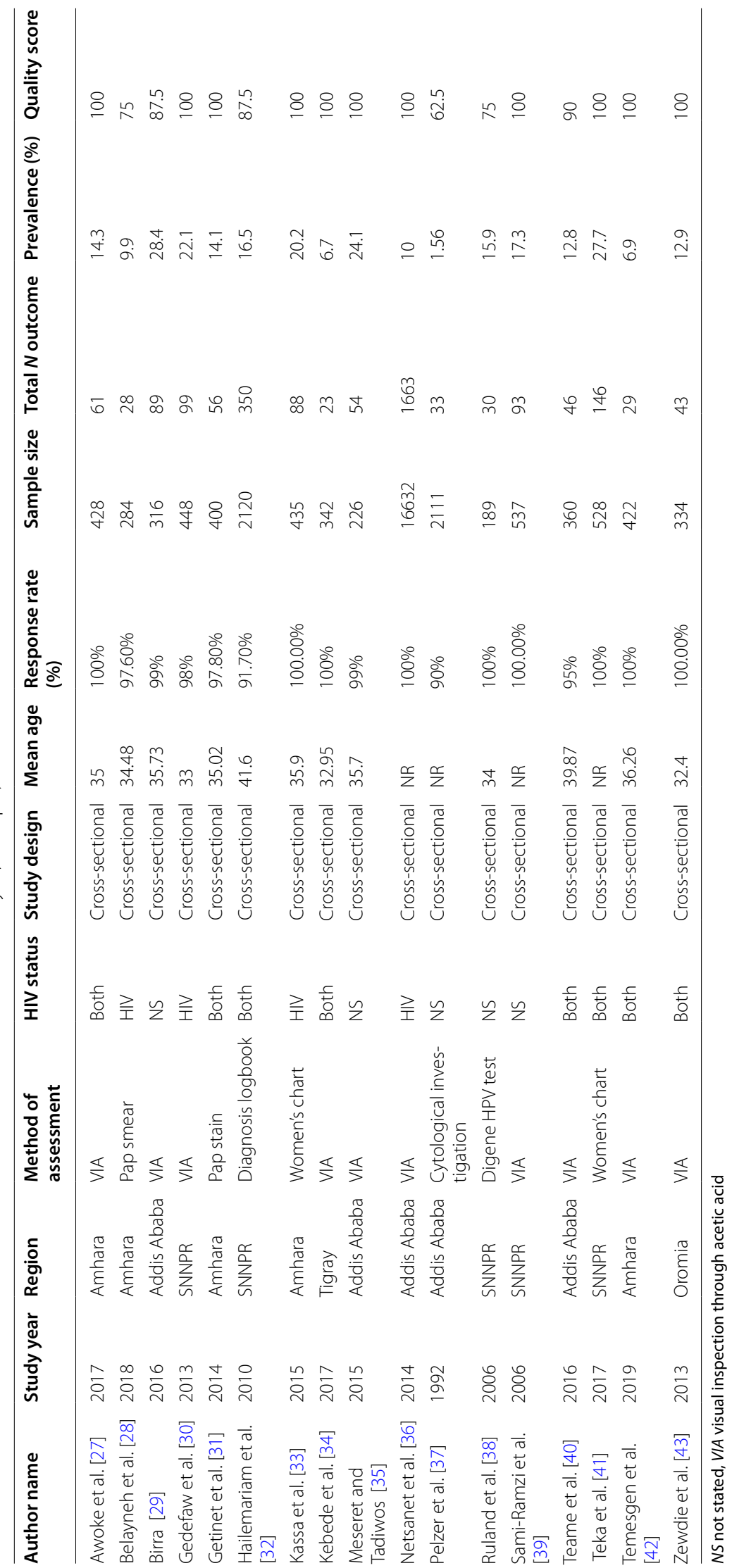




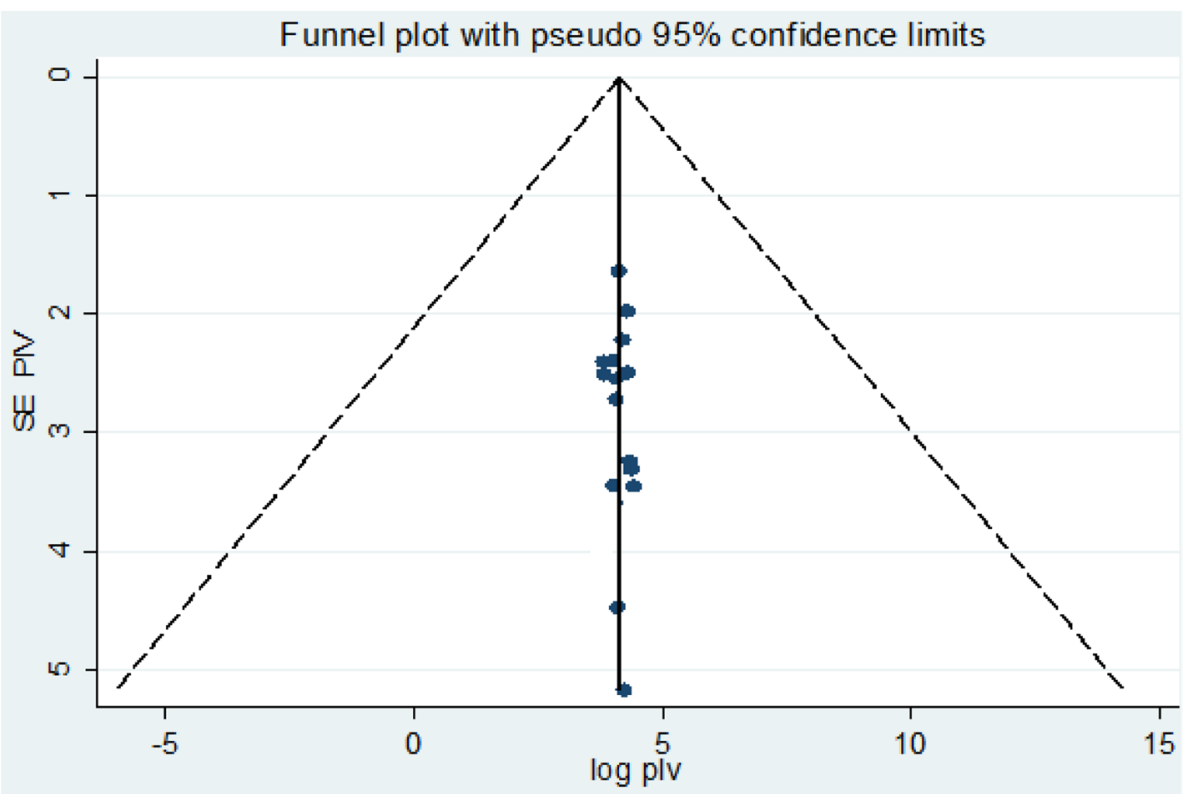

Fig. 2 Funnel plot presentation to assess publication bias for precancerous cervical lesion in Ethiopia: systematic review and meta-analysis, 2019

Ababa City Administration which the pooled prevalence was 15.10 (95\% CI 4.77-25.44).

An additional subgroup analysis was done using the HIV status of study participants. Accordingly, the highest pooled estimate of precancerous cervical lesion was found in those study participants whose sero-status is unknown, 17.27\% (95\% CI 5.12-29.41). However, the pooled prevalence rate of precancerous cervical lesion in those study participants whose status was confirmed to be HIV-positive was $15.27 \%$ (95\% CI 9.06-21.48) (Table 3).

The meta-regression analysis was conducted considering publication years, HIV status, sample size, and study area. However, the results showed that none of these variables was a statistically significant source of heterogeneity.

\section{Trend analysis}

Trends of the prevalence of precancerous cervical lesion were done to observe the patterns of the disease in Ethiopia using the reported prevalence and year of study. A significant upward trend in the prevalence of precancerous cervical lesion was observed from 1992 to 2019 ( $B$ $=0.56, P=0.014$ ) (Fig. 4).

\section{Discussion}

Promoting CC awareness and the importance of screening has been advocated in Ethiopia since 2008 [50]. Despite this fact, very few women receive screening services [51]. The current systematic review and meta-analysis aimed to estimate the pooled prevalence of precancerous cervical lesion among Ethiopian women between 1992 and 2019, providing information about trends and demographics. The result revealed that the pooled prevalence of precancerous cervical lesion was $15.16 \%$ (95\% CI 10.16, 19.70). This finding is almost consistent with a study done in Rural Nigerian women of which $16.6 \%$ had precancerous cervical lesion [52]. A facility-based cross-sectional study of mostly HIV+ women from Tanzania and another study from Nigeria revealed $17 \%$ and $17.8 \%$ women, respectively, had precancerous cervical lesion $[53,54]$.

Our meta-analysis shows higher prevalence than from a systematic review done in Tanzania that showed the overall prevalence of precancerous cervical lesion was 9.2\% [55] and a study from Zaria State in Nigeria in which the overall prevalence of precancerous cervical lesion was $4.8 \%$ [56]. Another study done in Kwara State, Nigeria, showed only $5 \%$ of study participants had precancerous cervical lesion [57]. A community-based screening in Turkey showed a precancerous cervical lesion prevalence of $9.4 \%$ precancerous cervical lesion [58]. A similar study in India using a community-based cervical cancer screening program among women of Delhi obtained $4.67 \%$ precancerous cervical lesion prevalence [59].

The possible explanation for this discrepancy might be the mean age of marriage among the respondents in the aforementioned studies was relatively higher. As early marriage is one of the risk factors for having precancerous cervical lesion, this might augment the incidence and 


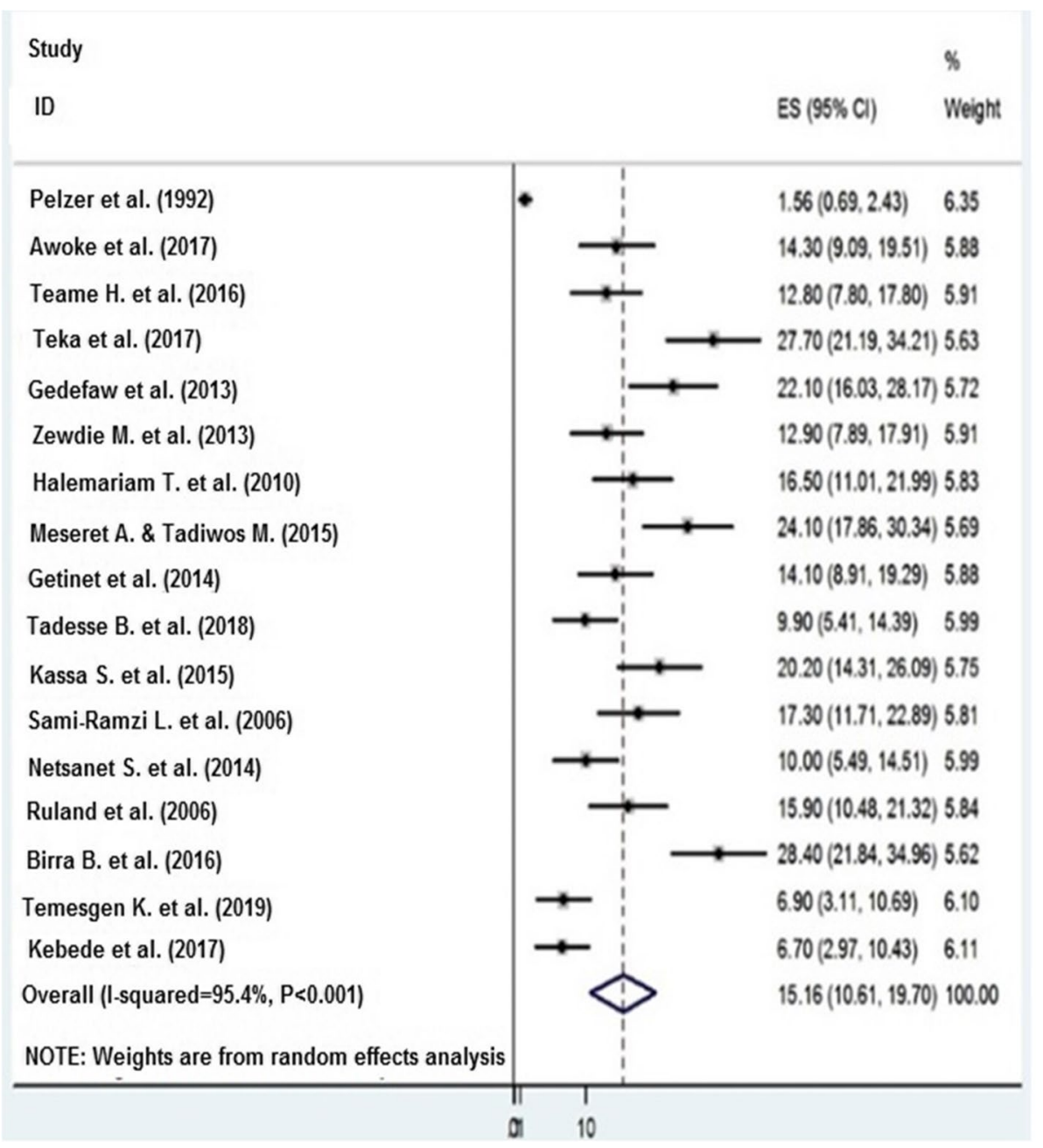

Fig. 3 Pooled prevalence of precancerous cervical lesion in Ethiopia, 2019

Table 3 Subgroup analysis showing the prevalence of precancerous cervical lesion among Ethiopian regions, 2019

\begin{tabular}{lllll}
\hline Variables & Region/city administration & Number of studies & Sample size & Estimate (95\% Cl) \\
\hline By region & Addis Ababa & 5 & 19,645 & $15.10(4.77,25.44)$ \\
& SNNPR & 5 & 3822 & $19.65(15.51,23.80)$ \\
& Amhara & 5 & 1969 & $12.76(8.36,17.16)$ \\
By HIV status & Other* & 2 & 676 & $9.56(3.51,15.62)$ \\
& Unknown/undiagnosed & 5 & 3379 & $17.27(5.12,29.41)$ \\
& Both (HIV+ and HIV-) & 8 & 4934 & $13.63(9.47,17.79)$ \\
Overall & HIV+ & 4 & 17,799 & $15.27(9.06,21.48)$ \\
\hline
\end{tabular}

prevalence of the precancerous cervical lesion. The other explanation might be most of researches conducted in the aforementioned countries were from urban settings and they might have awareness and access to information and this might lead them to have early screening for the disease.

This finding is lower than a study done in Senegal that revealed $21.03 \%$ of study participants had precancerous 


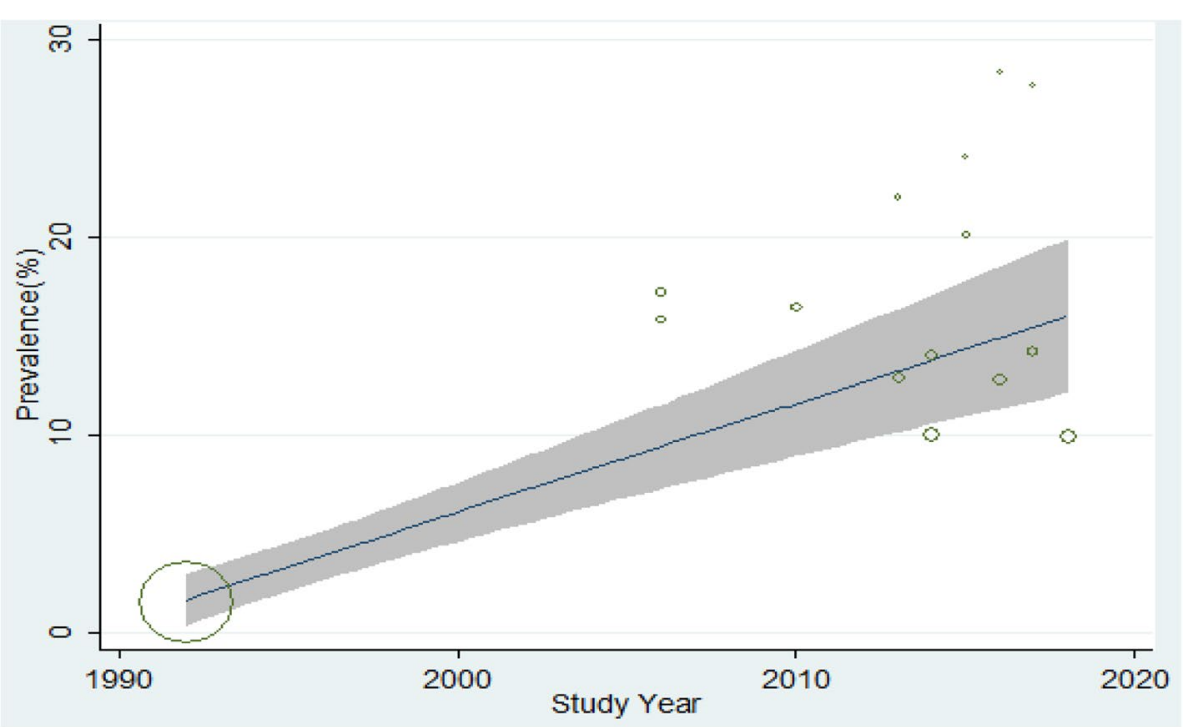

Fig. 4 Trends of precancerous cervical lesion in Ethiopia using prevalence and year of study $(B=0.56, P=0.014)$

cervical lesion [60]. Another study done in Nigeria among HIV-positive women showed that $22.2 \%$ of women had precancerous cervical lesion [61]. The possible elucidation for such discrepancy between the current finding and other comparable study findings might be due to the difference in the socio-demographic variations in the included study participants. A study from countries contained a data collected mostly from the rural population and HIV-positive women while in our study, both urban and rural settings were considered. The other possible explanation for the above variation could be due to the difference in study design.

Based on the subgroup analysis, the highest pooled prevalence of precancerous cervical lesion was seen in Southern Nation and Nationalities and People's Region (SNNPR) (19.65\%). This finding was higher compared to studies conducted in Addis Ababa and Amhara region that revealed $15.10 \%$ and $14.35 \%$, respectively. The possible explanations for this variation might be accounted by variations in information dissemination across the regions for reproductive health women about the disease. Hence, those women who had no information might remain with the symptom/disease. Understanding the reasons for these variations will include comparing regional methods of information dissemination related to reproductive health and disease. All of these can also be compared to interventions in countries with lower prevalence rates, in order to identify best practices, and promising interventions appropriate for implantation in Ethiopia.

It is known that HIV infection is one of the main risk factors for the development of cervical dysplasia. On this regard, there are many reports on the association of HIV with increased risk of cervical dysplasia [54, 61-63]. However, the current systematic review and meta-analysis in the subgroup analysis revealed that the highest precancerous cervical lesion (17.27\%) was found in those study participants whose sero-status was unknown. This finding is supported by the WHO report that stated the likely pattern of precancerous cervical lesion expected in a previously unscreened population of women [31, 33, 64, 65]. In addition to this, the possible explanation for this finding might be accounted by participants who were not screened for HIV might be exposed for other risk factors for cervical cancer. Moreover, though their sero-status was not known at the time of precancerous cervical lesion screening, they might previously know that their sero-status for HIV/ AIDS was positive.

\section{Limitation}

In the current systematic review and meta-analysis, we noticed the following limitations. The pooled prevalence which was determined using the eligible 17 studies only represented four different administrative regions in Ethiopia. Hence, it does not represent the whole country as studies in more than half of the regions were not eligible for inclusion in the current review. Utilization of various data collection tool in the included studies might have its own influence in generalizing the finding. In addition, potentially unpublished studies were not considered and latent sources 
of heterogeneity may also have an impact in the overall finding of the result.

\section{Conclusion}

The current study revealed that one in six study participants were positive for precancerous cervical lesion. The study also showed that there is an increased trend of precancerous cervical lesion prevalence in the country. Possible ways to increase awareness programs may include using various social media. In addition, best practices in achieving high vaccination coverage should be considered from other successful countries.

\section{Abbreviations}

CC: Cervical cancer; Cl: Confidence interval; HIV: Human immuno virus; HPV: Human papilloma virus; JBI: Joanna Briggs Institute; VIA: Visual inspection with acetic acid; VILI: Visual inspection with Lugol's iodine; SNNPR: Southern Nations Nationalities and People Region; WHO: World Health Organization.

\section{Acknowledgements}

We would like to extend our deep acknowledgment to Professor Barbara J. Engebretsen for proofreading our manuscript.

\section{Authors' contributions}

AS conceived and designed the research protocol. ASK, TD, and YB searched the studies and extracted all necessary data. GD, AE, and YW checked the data extraction process. AS and TD led the revision and edit the manuscript. All the rest authors BZ, EA, SK, GM, and WA involved in revising and editing the final version of the manuscript. All authors have read and approved the final draft of the manuscript.

\section{Funding}

No fund was obtained to carry out this study.

\section{Availability of data and materials}

All data are available in the manuscript.

\section{Declarations}

Ethics approval and consent to participate

Not applicable.

\section{Consent for publication}

Not applicable.

\section{Competing interests}

The authors declare that they have no competing interests.

\section{Author details}

${ }^{1}$ Department of Adult Health Nursing, College of Medicine and Health Sciences, Bahir Dar University, Bahir Dar, Ethiopia. ${ }^{2}$ Department of Pediatrics and Child Health Nursing, College of Medicine and Health Science, Bahir Dar University, Bahir Dar, Ethiopia. ${ }^{3}$ ALKAN Health Science Business and Technology College, Bahir Dar, Ethiopia.

Received: 30 April 2020 Accepted: 19 October 2021

Published online: 01 November 2021

\section{References}

1. American Cancer Society. Cancer facts \& figures. 2011. p. 4.

2. Kasa AS, Tesfaye TD, Temesgen WA. Knowledge, attitude and practice towards cervical cancer among women in Finote Selam city administration, West Gojjam Zone, Amhara Region, North West Ethiopia, 2017. Afr Health Sci. 2018;18(3):623-36.

3. Getahun F, Mazengia F, Abuhay MBZ. Comprehensive knowledge about cervical cancer is low among women in Northwest Ethiopia. BMC Cancer. 2013;13(2):1-7.

4. Eke NO, et al. Knowledge of cervical cancer and its screening amongst female workers in Nnewi, Nigeria. Afri Med J. 2010;1(1):1-6.

5. Adamu AN, et al. The effect of health education on the knowledge, attitude, and uptake of free Pap smear among female teachers in Birnin Kebbi, North-Western Nigeria. Niger J Clin Pr. 2012;15(3):15-23.

6. Desta $K$, et al. The effect of HIV coinfection, HAART and TB treatment on cytokine/chemokine responses to Mycobacterium tuberculosis (Mtb) antigens in active TB patients and latently Mtb infected individuals. Tuberculosis. 2016:96:131-40.

7. Mccarey C. et. Awareness of HPV and cervical cancer prevention among Cameroonian healthcare workers. BMC Womens Heal. 2011;11(45):1-7.

8. Ferlay J, et al. Estimates of worldwide burden of cancer in 2008. Int J Cancer. 2010;127:2893-917.

9. Anantharaman W. A cross-sectional study on knowledge, attitude, and practice on cervical cancer and screening among female health care providers of Chennai corporation. J Acad Med Sci. 2013;2(4):124-8.

10. Denny $L$, et al. Human papillomavirus infection and cervical disease in human immunodeficiency virus-1-infected women. ObstetGynecol. 2008;111:1380-7.

11. Wondimu YT. Cervical cancer: assessment of diagnosis and treatment facilities in public health institutions in Addis Ababa. Ethiopia. Ethiop Med J. 2015;53:65-74.

12. Package WHO. WHO : Cervical cancer. 2018. Available from: https://www. who.int/cancer/prevention/diagnosis-screening/cervical-cancer/en/

13. Wittet S. Cervical cancer prevention and the millennium development goals. Bull World Heal Organ. 2008;86:488-90.

14. Bailie RS, et al. Trends in cervical cancer mortality in South Africa. Int J Epidemiol. 1996;25:488-93.

15. Al FM. et. Breast and cervical cancer in 187 countries between 1980 and 2010: a systematic analysis. Lancet. 2011;378(7):1-10.

16. Denny L, Anorlu R. Cervical cancer in Africa. Cancer Epidemiol Biomarkers Prev. 2012;21(9):1434-9 Available from: http://globocan.iarc.fr/. and http://www.who.int/hpvcentre.

17. Louie KS, et al. Epidemiology and prevention of human papillomavirus and cervical cancer in sub-Saharan Africa: a comprehensive review. Trop Med Int Heal. 2009;14:1287-302.

18. WHO/ICO. Human papilomavirus and related cancers in Ethiopia: summary report. 2014.

19. Waktola EA, Mihret WBL. HPV and burden of cervical cancer in east Africa. GynecolOncol. 2005;99:201-2.

20. Jemal A. et. Cancer burden in Africa and opportunities for prevention. Cancer. 2009;118:4372-84.

21. Tesfa A. Combating cervical cancer in Ethiopia; 2015. p. 1-3.

22. World Health Organization. Human papilloma virus and related cancers in Ethiopia: summary report; 2009. p. 1-5.

23. Bruni $L$ et al.. ICO Information Centre on HPV and Cancer (HPV Information Centre). Human papillomavirus and related diseases in Ethiopia. Summary Report 2014. 2014. Available from: www.hpvcentre.net

24. Moher D, et al. Preferred reporting items for systematic reviews and meta-analyses: the PRISMA statement. PLoS Med. 2009;6(6):1000097.

25. The Joanna Briggs Institute. Checklist for analytical cross sectional studies; 2016. p. 3-4.

26. Aklilu E, Setegn E, Tesfa D. A systematic review and meta-analysis of vertical transmission route of HIV in Ethiopia. BMC Infect Dis. 2018;18:283.

27. Derbie A, Mekonen D, Mezgebu Y, Biadglegne F. Cervical lesion detection using visual inspection with acetic acid and associated factors among Ethiopian women. Ethiop Med J. 2019;57(2):1-9.

28. Belayneh T, Habtamu Mitiku FW. Precancerous cervical lesion and associated factors among HIV-infected women on ART in Amhara Regional State, Ethiopia: a hospital-based cross-sectional study. Int J Heal Sci Res. 2019;13(3):1-6.

29. Bedassa BB. Acceptability of cervical cancer screening using See and Treat (SAT) approach and determinant factors among women of reproductive age in health centers in Addis Ababa, Ethiopia: Addis Ababa University; 2017. 
30. Gedefaw A, Astatkie A, Tessema GA. The prevalence of precancerous cervical cancer lesion among HIV-infected the prevalence of precancerous cervical cancer lesion among HIV-infected women in Southern Ethiopia: a cross-sectional study. PLoS One. 2013;8(12):1-9.

31. Getinet M, Gelaw B, Sisay A, Mahmoud EAA. Prevalence and predictors of Pap smear cervical epithelial cell abnormality among HIV-positive and negative women attending gynecological examination in cervical cancer screening center at Debre Markos referral hospital, East Gojjam, Northwest Ethiopia. BMC Clin Pathol. 2015;15(16):1-10. Available from:. https:// doi.org/10.1186/s12907-015-0016-2.

32. Hailemariam T, Yohannes B, Aschenaki H, Mamaye EOG, et al. Cancer science \& therapy prevalence of cervical cancer and associated risk factors among women attending cervical cancer screening and diagnosis center at Yirgalem. J Cancer Sci Ther. 2017;9(11):730-5.

33. Kassa S, Dile M, Zenebe KBM. Precancerous lesions of cervix among women infected with HIV in Referral Hospitals of Amhara Region, Northwest Ethiopia: a cross sectional study. Afr Health Sci. 2019;19(1):1-10.

34. Kebede M, Belay SAH. Prevalence of precancerous cervical lesion and associated factors among women in North Ethiopia. J Public Heal Epidemiol Full. 2017:9(March):46-50.

35. Meseret ATM. Prevalence of via positive cervical lesions and determinant factors among women attending regular gynecology outpatient department (RGOPD) at Saint Paul's Hospital Millennium Medical College (SPHMMC). Ethiop J Reprod Heal. 2018;10(2):22-30.

36. Netsanet S, Salvador-Davila KK, Brooks M, Weldegebreal T, et al. The single-visit approach as a cervical cancer prevention strategy among women with HIV in Ethiopia: successes and lessons learned. Glob Heal Sci Pract. 2016;4(1):87-98.

37. Pelzer A, Duncan E, Tibaux G, Mehari L. A study of cervical cancer in Ethiopian women, vol. 35. Belgium: University of Liège; 1992. Available from: https://www.ncbi.n/m.nih.gov/pubmed/1511118

38. Ruland R, Prugger C, Schiffer R, Regidor M, Lelle RJ. Prevalence of human papilloma virus infection in women in rural Ethiopia. Springer. 2006;21:727-8.

39. SamiSami-Ramzi L-B, Prugger C, GH KM, et al. Cervical human papillomavirus prevalence and genotype distribution among hybrid capture 2 positive women 15 to 64 years of age in the Gurage zone, rural Ethiopia. Infect Agent Cancer. 2014;9(33):1-9.

40. Teame H, Addissie A, Ayele W, Hirpa S, Gebremariam A, et al. Factors associated with cervical precancerous lesions among women screened for cervical cancer in Addis Ababa, Ethiopia: a case control study. PLoS One. 2018;39:1-13.

41. Teka T, Kote M, Kejela GGT. Magnitude and factors associated with precervical cancer among screened women in Southern Ethiopia. Adv Public Heal. 2019;10:1-9.

42. Temesgen K, Workie A, Dilnesa TAM. Proportions of pre-cancerous cervical lesions and its associated factors among women clients in the age group of 30-49yrs in gynecology ward of Dessie Referral Hospital and FGAE. North-East. J Cancer Tumor Int. 2019;9(2):1-15.

43. Zewdie M, Tesfamichael AFA. Prevalence and factors associated with VIA positive result among clients screened at Family Guidance Association of Ethiopia, south west area office, Jimma model clinic, Jimma, Ethiopia 2013: a cross-sectional study. BMC Res Notes. 2015:8(618):1-7.

44. Huedo-Medina TB, et al. Assessing heterogeneity in meta-analysis: Q statistic or 12 index? Psychol Methods. 2006;11(2):193.

45. Giggings, Thompson D and A, Measuring inconsistency in meta-analyses. Br Med J. 2003;327(7414):557.

46. Borenstein $M$, et al. A basic introduction to fixed-effect and randomeffects models for meta-analysis. Res Synt Methods. 2010;1(2):97-111.
47. Rendina-Gobioff G. et al. Detecting publication bias in random effects metaanalysis: an empirical comparison of statistical methods. 2006.

48. Kelley GAKK. Statistical models for meta-analysis: a brief tutorial. World J Methodol. 2012;2(4):2012.

49. Jackson D, et al. How does the DerSimonian and Laird procedure for random effects meta-analysis compare with its more efficient but harder to compute counterparts? J Stat Plann Inference. 2010;140(4):961-70.

50. Bayu H, Berhe Y, Mulat A, Alemu A. Cervical cancer screening service uptake and associated factors among age eligible women in Mekelle Zone, Northern Ethiopia, 2015: a community based study using health belief model. PLoS One. 2016;11(3):1-13.

51. Gebremariam T. Human papillomavirus related cervical cancer and anticipated vaccination challenges in Ethiopia. Int J Health Sci (Qassim). 2016;10(1):137-43.

52. Schnatz PF, Markelova NV, Holmes D, Mandavilli SR, Sullivan DMO, Ph D. The prevalence of cervical HPV and cytological factors of rural Nigerian women. JWomen's Heal. 2008;17(2):1-7.

53. Obure J, Olola O, Swai B, Mlay P, Masenga G, Walmer D. Prevalence and severity of cervical squamous intraepithelial lesion in a tertiary hospital in northern Tanzania. Tanzan J Health Res. 2009;11(4):163-9.

54. Makurdi H, Swende TZ, Ngwan SD, Swende LT. Prevalence and risk factors for cervical squamous intraepithelial lesions among women infected with HIV-1 in Makurdi, Nigeria. Int J Women's Heal. 2012;4:55-60.

55. Runge AS, Bernstein ME, Lucas AN, Tewari KS. Gynecologic oncology reports cervical cancer in Tanzania: a systematic review of current challenges in six domains. Gynecol Oncol Reports, Available from. 2019;29(May):40-7 https://doi.org/10.1016/j.gore.2019.05.008.

56. Adekunle OO, MOAS. Letters to Editor Prevalence of cervical intraepithelial neoplasia in Zaria. Ann Afr Med. 2010;9(3):2010.

57. Durowade KA, Osagbemi GK, Salaudeen AG, Musa OI, Akande TM, Babatunde OA, et al. Prevalence and risk factors of cervical cancer among women in an urban community of Kwara State, North Central Nigeria. J Prev Med Hyg. 2012;53:213-9.

58. Aydin S, Erturk B KH. An example of community-based cervical cancer screening. 2010. Available from: www.ukdk.org/pdf/kitap/en/48.

59. Sharma P, Rahi MLP. A community-based cervical cancer screening program among women of Delhi using camp approach. IJCM. 2010;35:86-8.

60. Diop M. HPV and cervical cancer in Senegal; 2013.

61. Agaba PA, Thacher TD, Ekwempu CC, Idoko JA. Cervical dysplasia in Nigerian women infected with HIV. Int J Gynecol Obstet. 2009;107(2):99-102.

62. Anorlu RI. Prevalence of abnormal cervical smears among patients with HIV in Lagos, Nigeria. West Afr J Med. 2007;26(2):143-7.

63. Dim CC, et al. Prevalence of cervical squamous intraepithelial lesions among HIV-positive women in Enugu, South-eastern Nigeria. J Obstet Gynaecol (Lahore). 2011;31(8):759-62.

64. Durowade KA, Hospital FT, State E, Osagbemi G, Salaudeen AG, Musa $O$. Prevalence and risk factors of cervical cancer among women in an urban community of Kwara State, North Central Nigeria. J Prev Med Hyg. 2012;53:85-8

65. Prazzini F, et al. The epidemiology of female genital tract cancers. IJGC. 1997;7:169-81.

\section{Publisher's Note}

Springer Nature remains neutral with regard to jurisdictional claims in published maps and institutional affiliations. 